\title{
Propagation Waves in a Ladder of Coupled Simultaneous Oscillators
}

\author{
Saori Fujioka ${ }^{\dagger}$, Yang Yang ${ }^{\ddagger}$, Yoko Uwate ${ }^{\dagger}$ and Yoshifumi Nishio ${ }^{\dagger}$ \\ $\dagger$ Dept. Electrical and Electronic Eng., Tokushima University \\ 2-1 Minami-Josanjima, Tokushima, Japan \\ Email: \{saori, uwate, nishio\}@ee.tokushima-u.ac.jp \\ $\$$ The Institute of Artificial Intelligence and Robotics., Xi'an Jiaotong University \\ No. 28 Xianning-West-Road, Xi'an, China \\ Email: yyang@mail.xjtu.edu.cn
}

\begin{abstract}
In this study, we investigate a ladder of simultaneous oscillators with three or four $L C$ resonators coupled by inductors. By computer simulations, we observe a new type of waves where oscillation frequency or/and oscillation amplitude of each resonator changes according to the wave propagation. Further, we confirm that the oscillation amplitude of each resonator depend on its positions.
\end{abstract}

\section{Introduction}

In the natural fields, various synchronization phenomena exists. For example, firefly luminescence, swing of pendulums, cardiac heartbeat, and so on, are well known as synchronization phenomena. Oscillators containing a nonlinear resistor whose $v-i$ characteristics are described by fifth-power nonlinear characteristics are known to exhibit hard excitation [1][2]. Namely, the origin is asymptotically stable and an proper initial condition, which is larger than a critical value, is necessary to generate the oscillation. Such an oscillator is often called as hard oscillator or said to have hard nonlinearity. Two identical oscillators with hard nonlinearities coupled by an inductor are investigated by Datardina and Linkens [3]. They have confirmed that nonresonant double-mode oscillations, which could not occur for the case of third-power nonlinearity, were stably excited in the coupled system oscillators having hard nonlinearity. They have also confirmed that four different modes coexist for some range of parameter values; zero, two single-modes, and a double-mode. In 1954, Schaffner reported that an oscillator with two degrees of freedom could oscillate simultaneously at two different frequencies when the nonlinear characteristics are described by a fifth-power polynomial function [4]. Kuramitsu also investigated the simultaneous oscillations for three or more degrees case theoretically and confirmed the generation of simultaneous oscillation with three frequencies by circuit experiments [5]. The simultaneous oscillations are definitely one of the most common nonlinear phenomena observed in various higher-dimensional systems in the natural science fields. However, after their pioneering works, as far as the authors know, there have not been many researches clarifying the basic mechanism of the simultaneous oscillations except [6][7].

In the past studies, we investigated two or three simultaneous oscillators coupled by a resistor or inductors [8][9][10]. We could confirm that double-mode and simultaneous oscillations are generated at the same time from inductively coupled simultaneous oscillators. We also investigated the case that twenty simultaneous oscillators with two $L C$ resonators are coupled by inductors in a one-dimensional array [13]. By computer simulations of the circuit model, we discovered two kinds of waves; the first wave is a propagating change of phase states between two horizontally adjacent resonators from in-phase to antiphase or from anti-phase to in-phase, which is the same as the phase-inversion waves in [11][12]. the second wave is a propagating change of oscillation states from oscillation death to oscillation or from oscillation to oscillation death.

In this study, we investigate twenty simultaneous oscillators with three or four $L C$ resonators coupled by inductors in a one-dimensional array. By computer simulation, we discover a new type of propagation waves. In this wave, oscillation frequency or/and oscillation amplitude of each resonator changes according to the wave propagation. Further, we confirm that the oscillation amplitude of each resonator depend on its positions.

\section{Circuit Model}

The circuit model is shown in Fig. 1. In the circuit, twenty simultaneous oscillators with three $L C$ resonators are coupled by inductors $L_{C}$ and each simultaneous oscillator consists of a nonlinear negative resistor, whose $v-i$ characteristics are described by a fifth-power polynomial function as

$$
i_{R}(v)=g_{1} v-g_{3} v^{3}+g_{5} v^{5} \quad\left(g_{1}, g_{3}, g_{5}>0\right),
$$

and three resonators with different natural frequencies $\left(\sqrt{L_{1} C_{1}}, \sqrt{L_{2} C_{2}}\right.$ and $\sqrt{L_{3} C_{3}}$ ). The equations governing the coupled oscillators are described by the following differential equations including twenty nonlinear functions $i_{R j}$ 


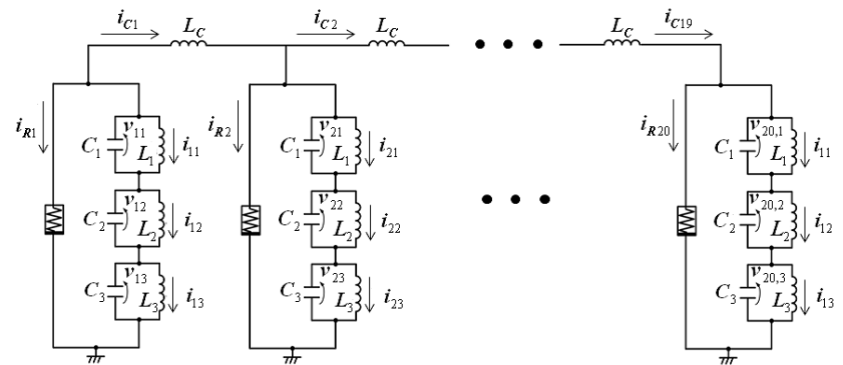

Figure 1: Twenty simultaneous oscillators with three resonators coupled by inductors in a one-dimensional array.

$(j=1,2, \cdots, 20)$

$$
\left\{\begin{array}{l}
C_{1} \frac{d v_{j 1}}{d t}=-i_{j 1}-i_{R j}-i_{C j}+i_{C, j-1} \\
C_{2} \frac{d v_{j 2}}{d t}=-i_{j 2}-i_{R j}-i_{C j}+i_{C, j-1} \\
C_{3} \frac{d v_{j 3}}{d t}=-i_{j 3}-i_{R j}-i_{C j}+i_{C, j-1} \\
L_{1} \frac{d i_{j 1}}{d t}=v_{j 1} \\
L_{2} \frac{d i_{j 2}}{d t}=v_{j 2} \\
L_{3} \frac{d i_{j 3}}{d t}=v_{j 3} \\
(j=1,2, \cdots, 20)
\end{array}\right.
$$

where $i_{C m}$ are the currents through the coupling inductors and are given as

$$
\begin{array}{rr}
i_{C m}=\frac{1}{L_{C}}\left\{L_{1}\left(i_{m 1}-i_{m+1,1}\right)\right. & +L_{2}\left(i_{m 2}-i_{m+1,2}\right) \\
& \left.+L_{3}\left(i_{m 3}-i_{m+1,3}\right)\right\} \\
(m=1,2, \cdots, 19), &
\end{array}
$$

with $i_{C 0}=i_{C, 20}=0$. The currents through the nonlinear resistors $i_{R j}$ are given as

$$
i_{R j}=i_{R}\left(v_{j 1}+v_{j 2}+v_{j 3}\right) \quad(j=1,2, \cdots, 20) .
$$

By using the following variables and parameters,

$$
\begin{aligned}
& v_{j k}=\sqrt[4]{\frac{g_{1}}{5 g_{5}}} x_{j k}, \quad i_{j k}=\sqrt[4]{\frac{g_{1}}{5 g_{5}}} \sqrt{\frac{C_{1}}{L_{1}}} y_{j k}, \\
& \alpha_{C 1}=\frac{C_{1}}{C_{2}}, \quad \alpha_{L 1}=\frac{L_{1}}{L_{2}}, \quad \gamma=\frac{L_{1}}{L_{C}}, \\
& \alpha_{C 2}=\frac{C_{1}}{C_{3}}, \quad \alpha_{L 2}=\frac{L_{1}}{L_{3}}, \\
& \varepsilon=g_{1} \sqrt{\frac{L_{1}}{C_{1}}}, \quad \beta=\frac{3 g_{3}}{g_{1}} \sqrt{\frac{g_{1}}{5 g_{5}}}, \quad t=\sqrt{L_{1} C_{1}} \tau \\
& (j=1,2, \cdots, 20) \quad(k=1,2,3),
\end{aligned}
$$

the normalized circuit equations are given as follows.

$$
\left\{\begin{array}{l}
\frac{d x_{j 1}}{d \tau}=-y_{j 1}-f\left(x_{j 1}+x_{j 2}+x_{j 3}\right)-y_{C j}+y_{C, j-1} \\
\frac{d x_{j 2}}{d \tau}=\alpha_{C 1}\left\{-y_{j 2}-f\left(x_{j 1}+x_{j 2}+x_{j 3}\right)-y_{C j}+y_{C, j-1}\right\} \\
\frac{d x_{j 3}}{d \tau}=\alpha_{C 2}\left\{-y_{j 3}-f\left(x_{j 1}+x_{j 2}+x_{j 3}\right)-y_{C j}+y_{C, j-1}\right\} \\
\frac{d y_{j 1}}{d \tau}=x_{j 1} \\
\frac{d y_{j 2}}{d \tau}=\alpha_{L 1} x_{j 2} \\
\frac{d y_{j 3}}{d \tau}=\alpha_{L 2} x_{j 3} \quad(j=1,2, \cdots, 20),
\end{array}\right.
$$

where $y_{C m}$ corresponding to $i_{C m}$ is given as

$$
\begin{array}{r}
y_{C m}=\gamma\left(y_{m 1}-y_{m+1,1}+\frac{y_{m 2}-y_{m+1,2}}{\alpha_{L 1}}+\frac{y_{m 3}-y_{m+1,3}}{\alpha_{L 2}}\right) \\
(m=1,2, \cdots, 19),
\end{array}
$$

with $y_{C 0}=y_{C, 20}=0$. The nonlinear function $f(\cdot)$, which corresponds to the $v-i$ characteristics of the nonlinear resistors, is given as

$$
f(x)=\varepsilon\left(x-\frac{\beta}{3} x^{3}+\frac{1}{5} x^{5}\right) .
$$

\section{Two kinds of waves [13]}

In our past study, we investigated the twenty simultaneous oscillators with two $L C$ resonators coupled by inductors and discovered coexisting two kinds of waves.

The first wave is a propagating change of phase states between two horizontally adjacent resonators from in-phase to anti-phase or from anti-phase to in-phase. An example of this type of waves is shown in Fig. 2. The vertical axes of this diagram are the sum of the voltages of two horizontally adjacent resonators. Hence, black area of the diagram shows in-phase synchronization states, while white area shows anti-phase synchronization states or oscillation death. From this figure, we can see that two phaseinversion waves propagates in the upper resonators, while all the lower resonators stop the oscillation.

The second wave is a propagating change of oscillation states from oscillation death to oscillation or from oscillation to oscillation death. An example of this type of waves is shown in Fig. 3. The vertical axes of this diagram correspond to the voltages of the resonators. Hence, white area simply shows the oscillation death. From this figure, we can confirm that the upper resonators oscillate only when the lower resonators stop to oscillate, and vice versa.

\section{Propagation wave in three $L C$ resonators}

In this study, we investigate the twenty simultaneous oscillators with three $L C$ resonators coupled by inductors in 


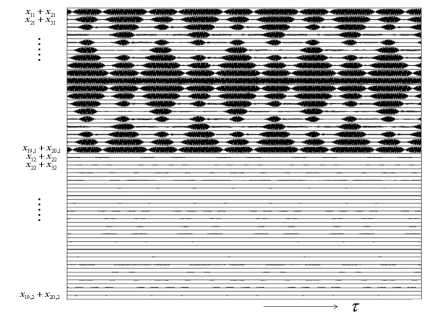

Figure 2: Propagating change of phase states between two horizontally adjacent resonators. $\alpha_{C}=0.57, \alpha_{L}=1.0, \gamma=0.01$, $\varepsilon=0.98$ and $\beta=2.73$.

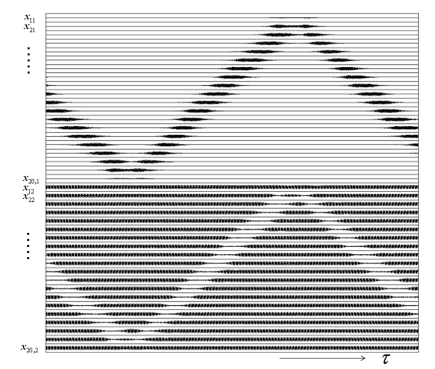

Figure 3: Propagating change of oscillation states. $\alpha_{C}=0.57$, $\alpha_{L}=1.0, \gamma=0.01, \varepsilon=0.98$ and $\beta=2.73$.

a one-dimensional array. We can observe a new type of wave propagation phenomenon which is different from the waves observed from the twenty simultaneous oscillators with two $L C$ resonators.

Figure 4 shows an example of the new propagation wave. The vertical axes of this diagram correspond to the voltages of the resonators; the top 20 graphs are the voltages of the upper resonators, the middle 20 graphs are the middle resonators, and the lower 20 graphs are the lower resonators.

There are two characteristics of this wave. First, all the resonators always oscillate, although the oscillation amplitude becomes smaller for the area without the new wave. This means that oscillation death cannot be observed. The second characteristic is that the propagation waves in the three region (upper, middle and lower) look like the same.

Furthermore, we can observe that the propagation speed of the waves depends on the coupling strength $\gamma$. Figure 5 shows how the propagation speed changes for different values of $\gamma$. The values of the other parameters of Fig. 5 are $\alpha_{C 1}=0.81, \alpha_{L 1}=1.0, \alpha_{C 2}=0.9, \alpha_{L 2}=1.0, \varepsilon=0.35$ and $\beta=3.3$. Moreover, if we change the value of parameter of $\alpha_{C}$ corresponding to the ratio of the capacitors, namely if we change the ratio of the natural frequencies of the resonators, the amplitude of some of the new waves becomes smaller as shown in Fig. 6. The values of the other parameters of Fig. 6 are $\alpha_{L 1}=1.0, \alpha_{L 2}=1.0, \gamma=0.2, \varepsilon=0.35$ and $\beta=3.3$.

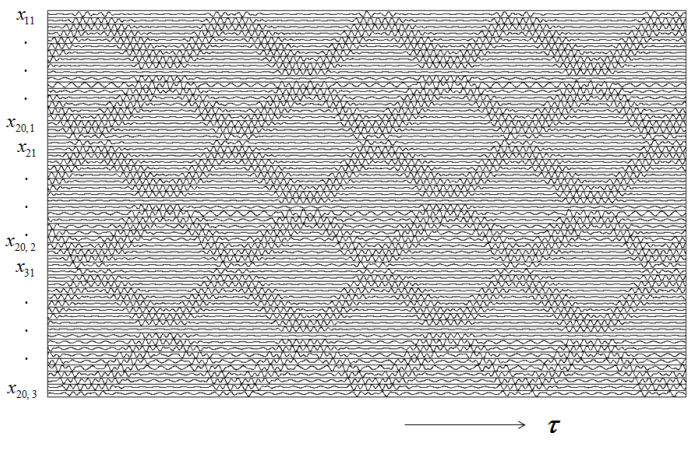

Figure 4: Propagation wave in three $L C$ resonators. $\alpha_{C 1}=0.81$, $\alpha_{L 1}=1.0, \alpha_{C 2}=0.9, \alpha_{L 2}=1.0, \gamma=0.3, \varepsilon=0.35$ and $\beta=3.3$.

\section{Propagation wave in four $L C$ resonators}

We also investigate the twenty simultaneous oscillators with four $L C$ resonators coupled by inductors in a onedimensional array. We can confirm the generation of the same propagation waves as those observed from the case of three $L C$ resonators. Figure 7 shows an example of the Propagation wave in four $L C$ resonators.

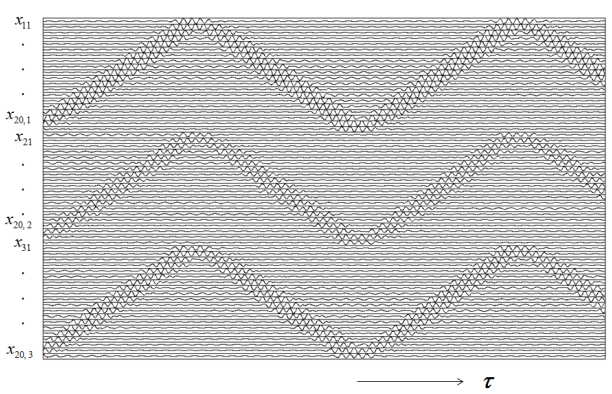

(a)

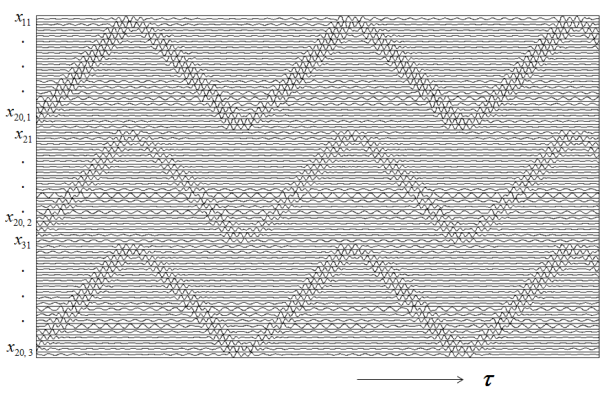

(b)

Figure 5: Propagation waves in three $L C$ resonators for different coupling strengths. (a) $\gamma=0.2$. (b) $\gamma=0.38$.

\section{Conclusions}

In this study, we investigated twenty simultaneous oscillators with three or four $L C$ resonators coupled by in- 


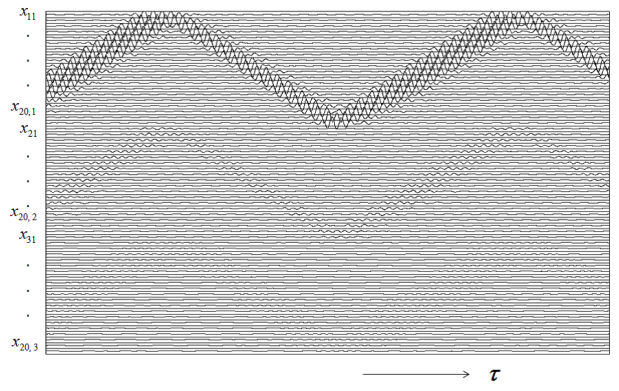

(a)

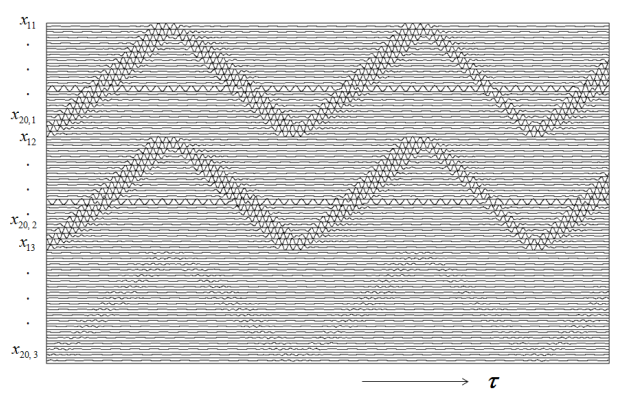

(b)

Figure 6: Propagation waves in three $L C$ resonators for different sets of natural frequencies. (a) $\alpha_{C 1}=0.1$ and $\alpha_{C 2}=0.3$. (b) $\alpha_{C 1}=$ 0.2 and $\alpha_{C 2}=0.9$.

ductors in a one-dimensional array. By computer simulation, we discovered a new type of propagation waves where oscillation frequency or/and oscillation amplitude of each resonator changed according to the wave propagation. Further, we confirmed that the oscillation amplitude of each resonator depended on its positions.

\section{Acknowledgment}

This work was partly supported by JSPS Grant-in-Aid for Scientific Research 22500203.

\section{References}

[1] C. Hayashi, Nonlinear oscillations in physical systems, Princeton Univ. Press, p. 367, 1984.

[2] V.I. Arnold, Geometrical methods in the theory of ordinary differential equations, Springer-Verlag, pp. 270-272, 1988.

[3] S.P. Datardina and D.A. Linkens, "Multimode oscillations in mutually coupled van der Pol type oscillators with fifth-power nonlinear characteristics," IEEE Trans. Circuits Syst., Vol. 25, No. 5, pp. 308-315, May 1978.

[4] J. Schaffner, "Simultaneous oscillations in oscillators," IRE Trans. Circuit Theory, Vol. 1, pp. 2-81, Jun. 1954.

[5] M. Kuramitsu and F. Takase, "Averaged potential analysis of multi mode oscillators with hard operating conditions,"

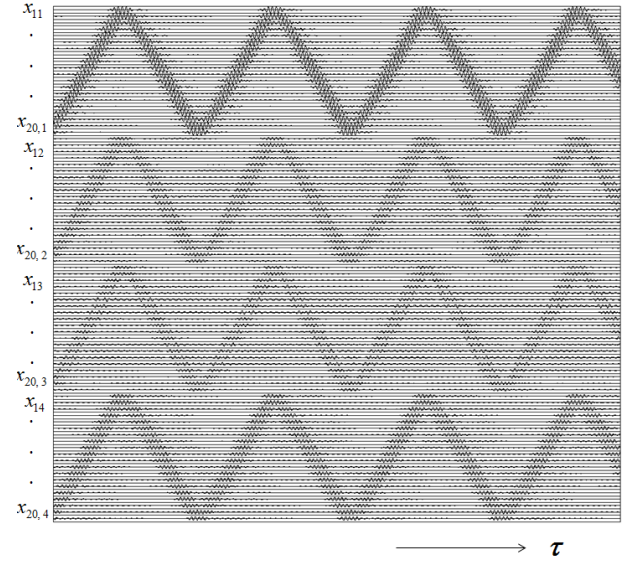

Figure 7: Propagation wave in four $L C$ resonators. $\alpha_{C 1}=0.65$, $\alpha_{L 1}=1.0, \alpha_{C 2}=0.71, \alpha_{L 2}=1.0, \alpha_{C 3}=0.81, \alpha_{L 3}=1.0, \gamma=0.2$, $\varepsilon=0.08$ and $\beta=3.3$.

IEICE Technical Report on NLP, Vol. 81, No. 13, pp. 1-10, Sep. 1981.

[6] M. Matsuki and S. Mori, "Asynchronous simultaneous oscillations in negative resistance oscillatory circuit containing periodically operating analog switch," IEICE Technical Report on CAS, Vol. 101, pp. 81-87, Jun. 1993.

[7] M. Matsuki and S. Mori, "Asynchronous excitation phenomena in negative resistance oscillatory circuit containing periodically operating analog switch," IEICE Technical Report on NLP, Vol. 94, pp. 51-58, May 1994.

[8] C. Higashi and Y. Nishio, "Investigation of synchronization phenomena in coupled simultaneous oscillators," Proceedings of NCSP'10, pp. 113-116, Mar. 2010.

[9] Y. Nishio, Y. Yang and Y. Uwate, "Synchronization phenomena in simultaneous oscillators coupled by an inductor," Proceedings of NCSP'11, pp. 147-150, Mar. 2011.

[10] S. Fujioka, Y. Yang, Y. Uwate and Y. Nishio, "Double-mode simultaneous oscillation in three coupled hard oscillators," Proceedings of NCSP'12, pp. 72-75, Mar. 2012.

[11] M. Yamauchi, M. Wada, Y. Nishio and A. Ushida, "Wave propagation phenomena of phase states in oscillators coupled by inductors as a ladder," IEICE Trans. Fundamentals, Vol. E82-A, No. 11, pp. 2592-2598, Nov. 1999.

[12] M. Yamauchi, Y. Nishio and A. Uahida, "Phase-waves in a ladder of oscillators," IEICE Trans. Fundamentals, Vol. E86-A, No. 4, pp. 891-899, Apr. 2003.

[13] S. Fujioka, Y. Yang, Y. Uwate and Y. Nishio, "Two kinds of waves in a ladder of coupled simultaneous oscillators," Proceedings of NDES'12, Jul. 2012 (printing). 